\title{
Article
}

\section{Non-Ionizing Millimeter Waves Non-thermal Radiation of Saccharomyces Cerevisiae - Insights and Interactions.}

\author{
Ayan Barbora ${ }^{1,3}$, Shailendra Rajput ${ }^{2}$, Konstantin Komoshvili ${ }^{3}$, Jacob Levitan ${ }^{3}$, Asher Yahalom ${ }^{2,4}$ \\ and Stella Liberman-Aronov ${ }^{1}$ \\ ${ }^{1}$ Department of Molecular Biology, Ariel University, Ariel, Israel \\ ${ }^{2}$ Department of Electrical \& Electronic Engineering, Ariel University, Ariel, Israel \\ ${ }^{3}$ Department of Physics, Ariel University, Ariel, Israel \\ ${ }^{4}$ Princeton University, Princeton, New Jersey 08543, USA \\ * Correspondence: Stella Liberman Aronov, Department of Molecular Biology, Ariel University, Kiryat \\ Hamada, POB 3, Ariel, Israel; Tel: (972) 3-937-1431; Fax: (972) 3-937-1422; E-mail: Stellar@ariel.ac.il
}

\begin{abstract}
Nonionizing millimeter-waves (MMW) interact with cells in a variety of ways. Here the inhibited cell division effect was investigated using 85-105 GHz MMW irradiation within the ICNIRP (International Commission on Non-Ionizing Radiation Protection) non-thermal $20 \mathrm{~mW} / \mathrm{cm}^{2}$ safety standards. Irradiation using a power density of about $1.0 \mathrm{~mW} / \mathrm{cm}^{2}$, SAR over 5-6 hours on 50 cells/ $\mu \mathrm{l}$ samples of Saccharomyces cerevisiae model organism resulted in $62 \%$ growth rate reduction compared to the control (sham). The effect was specific for $85-105 \mathrm{GHz}$ range, and was energy and cell density dependent. Irradiation of wild type and $\Delta$ rad52 (DNA damage repair gene) deleted cells presented no differences of colony growth profiles indicating non-thermal MMW treatment does not cause permanent genetic alterations. Dose versus response relations studied using a standard horn antenna $\left(\sim 1.0 \mathrm{~mW} / \mathrm{cm}^{2}\right)$ and compared to that of a compact waveguide $\left(17.17 \mathrm{~mW} / \mathrm{cm}^{2}\right)$ for increased power delivery resulted in complete termination of cell division via non-thermal processes supported by temperature rise measurements. We have shown that non-thermal MMW radiation has potential for future use in treatment of yeast related diseases and other targeted biomedical outcomes.
\end{abstract}

Keywords: Non-ionizing Radiation; Millimeter waves; Novel biomedical applications; Yeast; Noninvasive devices

\section{Introduction}

The influence of millimeter wave (MMW) radiation on biological systems has gained prominence in recent years because of two important reasons: 1 ) to establish safety standards for the use of MMWs in communication devices, 2) to understand the mechanisms of interaction between MMW and living systems. These investigations have opened new avenues for potential applications of MMW in the field of biomedical devices, for applications such as selective targeting of cancer cells. MMW in the range of $75-110 \mathrm{GHz}$ (W-band) are classed as nonionizing radiation because of the low 0.3-0.4 meV range of the energy of its photons.

Cancer, considered to be one of the deadliest human diseases, is challenging to diagnose at early stages [1]. Cancer is known to arise from accumulated mutations in oncogenes and tumor suppressor genes, leading to uncontrolled tumor cell growth $[2,3]$. Conventional radiation therapy in cancer treatment gives rise to many detrimental side effects [2] including the development of other more dangerous cancers due to the ionizing radiation (involved in such treatments) resulting in mutagenesis [3]. Thermal ablation techniques have been employed as the other alternative but with 
restricted application in order not to burn normal cells/tissues during treatment. Our experiments demonstrated that non-ionizing MMW (75-105 GHz) exposure with a non-thermal power density of $0.2 \mathrm{~mW} / \mathrm{cm}^{2}$ can elicit morphological changes in H1299 human lung cancer cells [4] leading to targeted apoptosis and mortality [5] without harming normal cells under the same exposure conditions. MMWs are also reported to be helpful for detecting different types of cancers [6]. Further, such technologies involving non-ionizing radiation has shown promising applications in the treatment of other diseases [7, 8] like gastrointestinal disorders, wound healing, remote monitoring of wounds, non-invasive detection of glucose levels, pain relief, diabetes, dermatitis, etc. However, the exact mechanism of the therapeutic effects on biological specimens is not well understood hindering the wide-scale application of this technology.

Being the simplest eukaryotic organism with a nucleus, Saccharomyces cerevisiae yeast cells are a standard model organism for in-vitro studies of cell division applicable up to higher eukaryotes. Many essential cellular processes in yeast and humans are similar, making yeast a suitable system to study basic molecular processes $[3,9,10]$. About $23 \%$ of the yeast genome is conserved in human cells, including all the corresponding biological functions and biochemical pathways remaining the same [11]. Characteristics of tumor cell growth have been discovered using models of yeast cell division $[3,9,10]$. Yeast cells are a cheap laboratory model that are simple to grow, culture, and experiment making it easier to unravel molecular mechanisms of MMW interactions within cells. Previous studies involving irradiation of wild type Saccharomyces cerevisiae yeast culture in aqueous suspensions have been highly ambiguous [12, 13]. These studies reported either no change or increased/decreased rate of growth upon microwave irradiation of $42 \mathrm{GHz}$ and $50 \mathrm{~mW}$ power [12, 13]. The authors reported the exclusion of thermal effects in such procedures by continuously monitoring temperature during the duration of exposure. On the other hand, MMW irradiation of yeast cells in the range of $41.650-41.798 \mathrm{GHz}$ for $4 \mathrm{~h}$ and $20 \mathrm{~mW}$ power found frequency sensitive results with increased cell growth at some frequencies and reduced at other values [14]. Another study confirmed the increased growth rate of yeast upon irradiation with $968 \mathrm{MHz}$ for $7 \mathrm{~h}$ at $17 \mathrm{dBm}$ power [15]. Results of such studies on the interaction of millimeter waves with biological samples are often met with inconsistence and non-reproducibility due to missing investigations of deeper biological mechanisms like influence on genetic material, the involvement of free radicals in oxidative processes, other metabolic disturbances etc. to correlate with the observed effects [16]. Thus, understanding the mechanisms of action will enable better use of MMW technology for therapeutic applications.

Given the rising challenges of biochemical drug resistance and the adverse effects from ionizing electromagnetic spectra limiting the use of conventional radiation therapies [3], molecular mechanisms of non-ionizing radiation procedures can suggest novel approaches to finding effective solutions. While non-ionizing radiation has mostly been used in thermal ablation procedures, we explored the effect of MMW (85-105 GHz) irradiation on the Saccharomyces cerevisiae yeast model of eukaryotic cell division in this article within the non-thermal safety standards set by the International Commission on Non-Ionizing Radiation Protection (ICNIRP) maintained for the stringency of analyses to unravel novel mechanisms. The MMW were propagated using a standard pyramidal horn antenna in one case and a standard wave guide in another. Radiated power density and power distribution across the aperture was analyzed and the influence of MMW exposure on yeast cells manifesting in the retarded cell growth effect studied. Irradiation of cells with deletion of the RAD52 gene ( $\Delta$ rad52), responsible for DNA damage repair was examined for radiation related genomic perturbations. Temperature rise measurements were conducted for analysis and maintenance of nonthermal exposure conditions. Non-ionizing MMW radiation absorption by cellular water content was investigated. Dosage dependent effects to achieve complete termination of yeast cell division were studied and compared using a waveguide delivering higher energy; which presented novel avenues for microbial infection control.

\section{Results}

2.1. MMW irradiation effects are frequency, energy dose and cell density dependent 
Wild Type (WT) Saccharomyces cerevisiae cells cultured under standard physiological conditions were subjected to 85, 95, $105 \mathrm{GHz}$ MMW exposures using a horn antenna and the effects compared with that of (sham) control. Cell densities $(10000,1000,100$ and 50 cells/ $\mu \mathrm{l})$ were calibrated to power dosages and treatment durations $(1,2,3,4,5$ and 6 hours) to unravel the threshold exposure conditions (ref. methods) required to elicit the inhibited cell division effect. Subsequently, six separate yeast colonies at the calibrated cellular density (50 cells/ $\mu \mathrm{l})$ and exposure duration (6 hours) were irradiated corresponding to each discrete frequency regime. The growth rate and division of treated yeast cells were then examined by incubating them under physiological conditions. 85-105 GHz MMW irradiation at the range 3.48-2.08 W/kg and power densities of $0.83-1.4 \mathrm{~mW} / \mathrm{cm}^{2}$ for 6 hours of exposure affected the growth rate of $(50$ cells $/ \mu \mathrm{l})$. WT yeast cells for all the examined frequencies and reduced the rate of division by up to $62 \%$ as compared to sham (control) (Fig. 1 and Table 1). Delay in cell proliferation becomes significantly noticeable over 3-5 hours of physiological incubation postirradiation treatment (Fig. 1a). Interestingly, although increasing the value of frequency is correlated with a reduction in respective power densities (ref. Table 1); the effect on cell proliferation was similar for each discrete frequency. This suggests that anti-proliferative MMW radiation effects was not affected by the amount of power density within the $85-105 \mathrm{GHz}$ range with a constant cell density (50 cells $/ \mu \mathrm{l}$ ) as long as a minimal threshold was attained. Indeed, a minimal power density of $0.83 \pm$ $0.02 \mathrm{~mW} / \mathrm{cm}^{2}$ at $105 \mathrm{GHz}$ (ref. Table 1) was sufficient to inhibit cell growth and division for $50 \mathrm{cells} / \mu \mathrm{l}$ samples of WT Saccharomyces cerevisiae.

Subsequently, the effect of different durations of irradiation ( $5 \mathrm{~h}$ versus $6 \mathrm{~h}$ ) and power densities ( $5 \mathrm{dBm}$ versus $6 \mathrm{dBm}$ ) was examined using constant frequency of $105 \mathrm{GHz}$ and constant energy. Using the same number of cells, the experiment demonstrates that the inhibited cell division effect was MMW dependent (Fig. 1b); being absent in the control (sham). We did not observe difference in the delay of the proliferation rate between 5 hours (with $6 \mathrm{dBm}$ power) and 6 hours (with $5 \mathrm{dBm}$ power) of irradiation we conjecture that this is so because both exposure regimes involved the same amount of energy (i.e. product of time and power) (ref. Fig. 1b). Single frequency at $105 \mathrm{GHz}$ was used as it affects WT yeast cell growth to the same extent as for all the other examined MMW frequencies (ref. Fig 1a); but provides a lower bound for power densities (ref. Table 1) to elicit the targeted outcome. The agar layer thickness was kept constant throughout this and other repeated experiments under similar conditions. Power densities emitted by the horn antenna aperture are presented in Table 1.
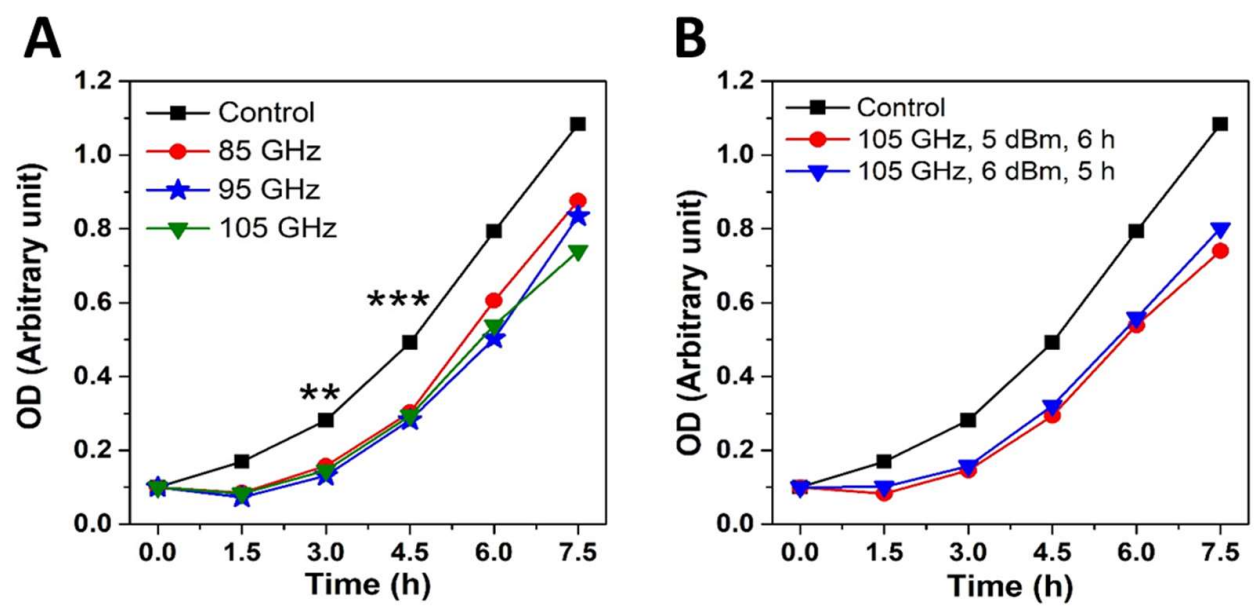

Figure 1. (A) Growth profiles of BY4741 Saccharomyces cerevisiae (50 cells/ $\mu$ l) cells measured in OD units after irradiation for 6 hours at $5 \mathrm{dBm}$. Plots indicate mean values of growth rate of six separate yeast colonies $(n=6)$ for each discrete frequency regime; Single Factor Analysis of variance (ANOVA) analysis $\left(^{* *}\right.$ indicates p-value $<0.01$ and ${ }^{* *}$ indicates p-value less than $\left.<0.001\right)$ (B) Growth profiles of BY4741 Saccharomyces cerevisiae (50 cells/ $\mu$ l) cells subsequent to irradiations of different time durations and power densities for a constant frequency (and constant energy) at $105 \mathrm{GHz}$. 


\begin{tabular}{cccc}
\hline Frequency $(\mathbf{G H z})$ & Power density $\left(\mathbf{m W} / \mathbf{c m}^{\mathbf{2}}\right)$ & $\mathbf{S A R}(\mathbf{W} / \mathbf{k g})$ & Time of irradiation $(\mathbf{h})$ \\
\hline 85 & $1.39 \pm 0.03$ & 3.38 & 6 \\
\hline 95 & $1.04 \pm 0.02$ & 2.61 & 6 \\
\hline 105 & $0.83 \pm 0.02$ & 2.08 & 6 \\
\hline 105 & $0.83 \pm 0.02$ & 2.08 & 5 \\
\hline
\end{tabular}

Table 1. Average energy flux at the aperture of the antenna for different frequencies (see an explanation and calculation in material and methods)

\subsection{Non-thermal MMW wave irradiation does not cause genomic DNA double-strand alterations}

The effects of MMW irradiation were observed to be persistent even beyond $3-6$ hours after termination of MMW exposure on the irradiated cells across six separate experiments. Evidently, thermal effects can be ruled out as the same non-thermal threshold powers were involved as reported earlier [17]. Irradiations of MMW under $1 \mathrm{~mW} / \mathrm{cm}^{2}$ are deemed not to give rise to thermal effects in living cells [14]. As our experiments involved power densities around $1 \mathrm{~mW} / \mathrm{cm}^{2}$ placing the results in a non-thermal range (also demonstrated in experiments later in this article), it is necessary to investigate other possible mechanism(s) responsible for the decreased growth rate of MMW irradiated cells. Therefore, the next step of the investigation was to check for the possible presence of genomic alterations arising due to radiation in the treated cells. In this direction, we examined for genomic DNA perturbations using the $\Delta$ rad52 deletion strain. Rad52 is a protein required to repair DNA double-strand breaks. Rad52p mediates Rad51p function in homologous recombination repair (HRR) in both yeast Saccharomyces cerevisiae and in mammalian cells of mice and humans [18]. Double-strand breaks (DSBs) are one of the most dangerous forms of DNA damage and are known to cause gross chromosomal rearrangements which are hallmarks of human cancers [19]. Yasuhara et al. highlighted the importance of human RAD52 for maintaining genome integrity [20]. In the absence of this protein in the $\Delta$ rad52 deletion strain, yeast cells reportedly die upon irradiation by DNA damaging electromagnetic spectra [21,22]. Irradiation of WT and $\Delta$ rad52 at the minimal threshold powers of $5 \mathrm{dBm}\left(0.83 \pm 0.02 \mathrm{~mW} / \mathrm{cm}^{2}\right)$ and frequency (105 GHz) for 5 and 6 hours respectively as indicated from the previous experiment did not yield any differences in colony growth profiles between the two (ref. Figure 1b). To remove the possibility that low power (corresponding to $0.83 \pm 0.02 \mathrm{~mW} / \mathrm{cm}^{2}$ surface power density) at $105 \mathrm{GHz}$ (ref. Table 1 ) was unable to unravel any differences in growth profiles both WT and $\Delta$ rad52 cells were subsequently exposed to $90 \mathrm{GHz}$ MMW at $5 \mathrm{dBm}$ power (corresponding to $\sim 1.0 \mathrm{~mW} / \mathrm{cm}^{2}$ surface power density) (ref. Table 1) for 6 hours and subsequently incubated under physiological conditions for analyses of serially diluted colony growth profiles. It was observed that both types of cells showed similar colony growth profiles (Fig. 2) even after serial dilutions were made to unravel any low intensity differences which may have been overlooked due to cell numbers; demonstrating that reduced cell growth is not accountable to genetic DNA damage of the treated cells. The absence of Rad52p didn't show any significant effect on the cells' growth after treatment indicating non-ionizing MMW radiation does not cause direct permanent genomic DNA double strand breaks/alterations, while other indirect DNA alterations are possible through regulatory protein networks as discussed later in the article. 


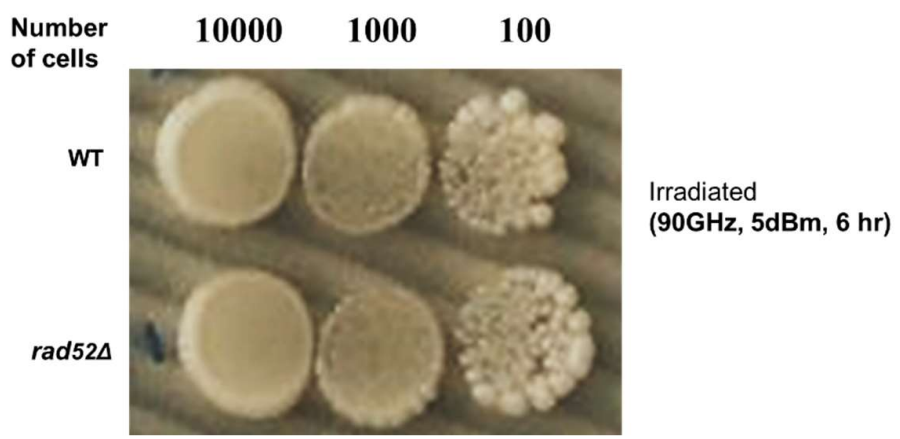

Figure 2. Representative image of the colony growth profiles of WT and $\Delta$ rad52 cells with serial dilutions after $(90 \mathrm{GHz}) \mathrm{MMW}$ irradiation at $5 \mathrm{dBm}$ for 6 hours. The cells were grown under physiological conditions at $30^{\circ} \mathrm{C}$, over 3-4 days post-treatment period. Number of cells after serial dilutions are as indicated. Five independent experiment were performed. No statistically significant differences were found between the two examined strains.

\subsection{MMW interaction with water as a factor for reduced cell growth}

In the above section, the experiment indicated that the MMW irradiation can affect cell growth/division without causing directed DNA molecules damage. However, the radiation may elicit perturbations of DNA regulatory proteins responsible for structural or chemical modifications of the genome and proteins regulating other metabolic processes. This can happen by interaction of the radiation with water which is known well absorb electromagnetic radiation in the microwave and infrared spectrum [23], which indirectly can affected protein structure and function.

Thus, we examine the amount of MMW irradiation which is solely absorbed by the yeast cells and the part that is reflected. Based on the energy penetrating the cells we may be able to unravel the contributions of MMW interactions with the cytosolic part of the cells, which contains many types of macromolecules. Therefore, we measured the absorbed power over time, represented as a ratio of incident and reflected powers for yeast cells spotted on SC agar. This was compared to that of plain water and blank SC agar without cells respectively. The irradiation conditions were kept constant at $85 \mathrm{GHz}$ for 5 hours $(5 \mathrm{dBm}$, at the maximal power level available to us corresponding to $1.39 \pm 0.02$ $\mathrm{mW} / \mathrm{cm}^{2}$ ) for stringency of analysis.

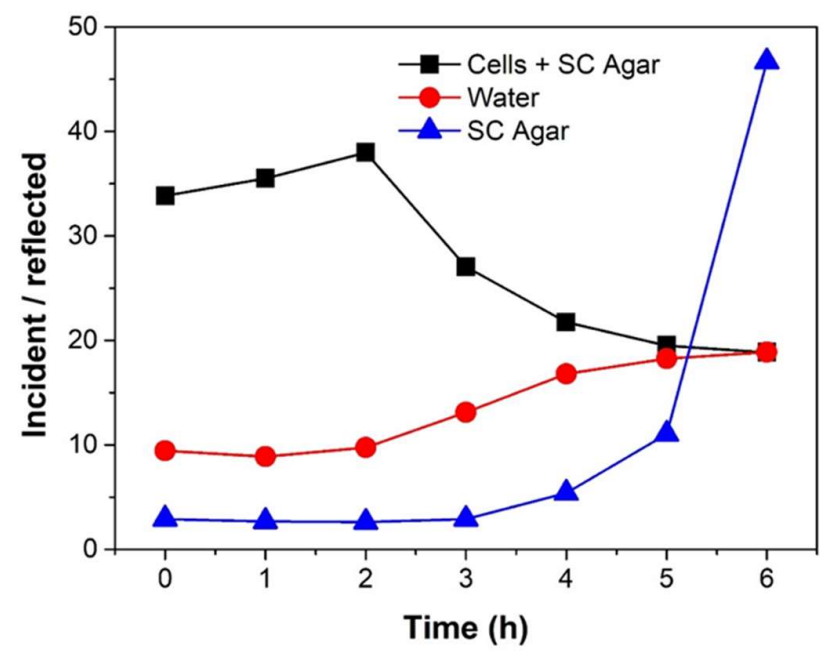


Figure 3. Absorbed power represented as a ratio of incident and reflected power from yeast cells spotted on Synthetic Complete (SC) agar compared to that of plain water and Synthetic Complete (SC) agar without cells respectively. Experiments were performed on a $1 \mu \mathrm{l}$ spot of 50 cells/ $\mu \mathrm{l}$ concentration with a frequency of $85 \mathrm{GHz}$ at an amplitude of $5 \mathrm{dBm}$ over 5 hours (corresponding to $1.39 \pm 0.02 \mathrm{~mW} / \mathrm{cm}^{2}$ surface power density).

The sample(s) absorbs a part of the incident radiation and the remaining is reflected. We find that the yeast cells spotted on SC agar absorbed more power (Fig. 3) as compared to plain water and blank SC agar without cells on the same volume of media used. Interestingly, plain SC agar reflected most of the incident power. The results suggest that non-thermal exposure of MMW could affect the proteins present in the cytosolic part of the cells through interactions with water molecules without involving heat shock proteins [24]. This could account for the observed phenomenon of cell growth inhibition without genetic perturbation or by damage of other functional cytosolic protein molecules and/or structures (ref. Fig. 2). The experiment also indicates that cells have a high absorbance of MMW irradiation by macromolecules within a cytosol made mostly of water separated from the environment within a confined volume of membrane bound structures.

Therefore, in order to ascertain any relation behind MMW absorption, temperature and volume of sample treated as indicated from the previous experiment, different volumes of water were irradiated using the horn antenna and temperature rise monitored. Irradiation conditions of frequency, power and duration of exposure were kept constant as mentioned previously to maintain empirical stringency. A $2^{\circ} \mathrm{C}$ rise of temperature measured using a digital thermometer was associated with a 6 hours exposure duration at $1.39 \mathrm{~mW} / \mathrm{cm}^{2}$ from a horn antenna (Fig. 4a) on a large volume of water $(6500 \mu \mathrm{l})$. Reduction in the size of the irradiated sample to a smaller volume of water $(250 \mu \mathrm{l})$ led to a $1^{\circ} \mathrm{C}$ increase in the rate of temperature rise (Fig. 4b). Interestingly, exposure involving a waveguide (delivering a higher power density of $17.17 \mathrm{~mW} / \mathrm{cm}^{2}$ ) on the same small volume of water $(250 \mu \mathrm{l})$ did not change the rate of temperature rise any further (Fig. 4c). Further, contrary to the expectation of the waveguide causing thermal ablation due to higher power density, the experiment demonstrates that thermal effects were practically absent in our irradiation setup. Therefore, under conditions of constant frequency, power and exposure duration; the radiation associated rise in temperature is inversely proportional to the volume of the sample irradiated. Finally, this experiment also confirms the hypothesis that biological cells exhibit high absorbance of MMW irradiation accountable to being constituted of water within a confined volume separated from the environment. The experiment demonstrates that MMW irradiation under the listed parameters raises temperatures up to $22{ }^{\circ} \mathrm{C}$ and is therefore not expected to elicit thermal stress on yeast cells which grow at physiological temperatures of $30^{\circ} \mathrm{C}$. 


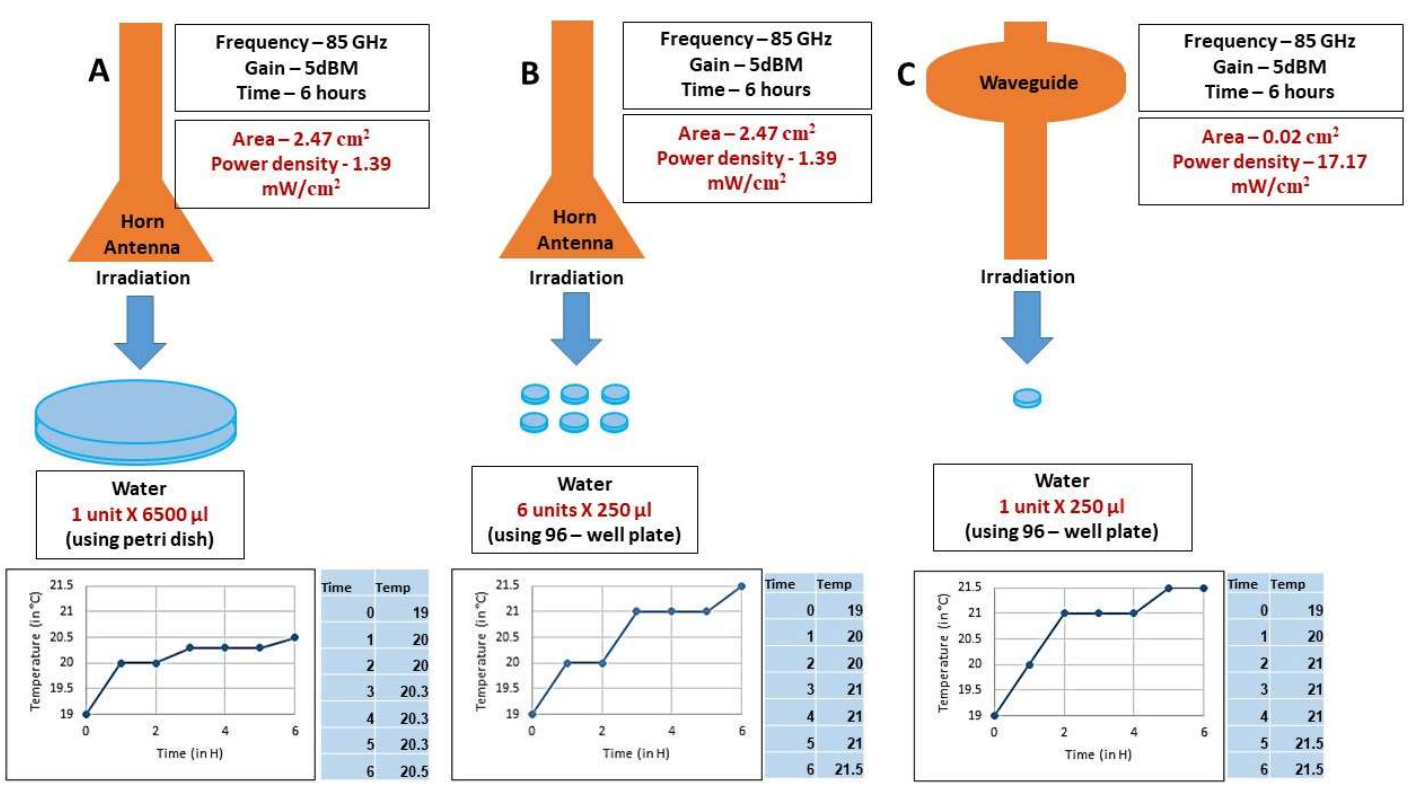

Figure 4. Comparison of temperature rise during $85 \mathrm{GHz}$ MMW exposure at constant power and treatment duration, involving horn antenna and wave guide. Indicated volumes of water were irradiated as illustrated. (A) Temperature rise of $6500 \mu \mathrm{l}$ water during MMW exposure at $1.39 \mathrm{~mW} / \mathrm{cm}^{2}$. (B) Temperature rise of $250 \mu \mathrm{l}$ water during MMW exposure at $1.39 \mathrm{~mW} / \mathrm{cm}^{2}$. (C) Temperature rise of $250 \mu \mathrm{l}$ water during MMW exposure at $17.17 \mathrm{~mW} / \mathrm{cm}^{2}$. Frequency, power and duration of exposure were kept constant at the values and specific experiments as indicated.

A wave-guide provides a focused beam of irradiation as compared to a horn antenna (ref. Fig. 4) allowing more precise energy delivery. To investigate potential applications of this exposure regime we performed MMW irradiation at $85 \mathrm{GHz}$ and $5 \mathrm{dBm}$ on BY4741 Saccharomyces cerevisiae WT yeast cells using an open-ended waveguide (Fig. 5). Single frequency at $85 \mathrm{GHz}$ was used as it provides the maximal threshold powers and affects WT yeast cell growth to the same extent as for all the other examined MMW frequencies (ref. Fig 1a). The thickness of agar medium and the number of cells were kept the same $(50 \mathrm{cells} / \mu \mathrm{l})$ as those mentioned in the previous experiments. Cells were treated for 3-4 hours at the non-thermal exposure power density of $17.17 \mathrm{~mW} / \mathrm{cm}^{2}$ (ref. Fig. 4). Subsequent incubation of these cells under physiological conditions (at $30^{\circ} \mathrm{C}$ for 2 days over a week) did not yield any colony growth. The experiment demonstrates that MMW irradiation using an openended waveguide at $\sim 12$ time's stronger power density than the one involving a horn antenna (ref. Fig. 1, 4 and Table 1) completely terminates cell growth and division.

\section{Discussion}

In this work, we showed that WT Saccharomyces cerevisiae cells in response to MMW irradiation $(85-105 \mathrm{GHz})$ at $\sim 1.0 \mathrm{~mW} / \mathrm{cm}^{2}$ for 6 hours using a standard pyramidal horn antenna reduced the rate of division by up to $62 \%$ as compared to sham (control) (ref. Fig. 1 and Table 1 ). The inhibition of the cell growth was performed for a constant cell density (50 cells/ $\mu \mathrm{l}$ sample). We studied the frequency and energy dose dependence over the tested range. Our results have showed a strong inhibition of proliferation as compared to previous published (contradictive) results using radiation in the MMW ranges and the specified conditions, which were mentioned in the introduction [12-15]. Antiproliferative effects of MMW radiation was elicited with a minimal power density of $0.83 \pm 0.02$ $\mathrm{mW} / \mathrm{cm}^{2}$ at $105 \mathrm{GHz}$ (Table 1) and was sufficient to inhibit cell growth and division (Fig. 1a) on the concentration of cells used (50cells $/ \mu \mathrm{l}$ ). Additionally, the effect was energy dose dependent as $5 \mathrm{dBm}$ for 6 hours at a constant frequency of $105 \mathrm{GHz}$ showed similar cell growth reduction as $6 \mathrm{dBm}$ for 5 hours (Fig. 1b). 
Next, we examined a hypothesis whether the MMW cell growth inhibition effect was due to permanent DNA genotoxic effects. Using the yeast strain with deletion of Rad52p, which is responsible for DNA double strand breaks repair, we found no significant effect on the cells' growth after irradiation treatment under the specified parameters (Fig.2). Our results indicate that growth inhibitory effect on WT yeast cells by non-ionizing MMW radiation does not occur due to permanent genomic alterations, while other indirect genomic effects may be possible through protein regulatory networks.

In general, non-ionizing radiations with low photon energies of $0.3-0.4 \mathrm{meV}$ are not expected to biochemically alter physiological DNA structure or function. Edwards et al. proposed a mechanism of coherent frequency-specific deposition of microwave energy on DNA in water [25]. The resonance of DNA molecules with irradiated spectra can be calculated in terms of the absorption coefficient. 2734 base pairs (bp) supercoiled circular DNA, 2734 bp linear DNA, 1786 bp linear DNA, and 948 bp linear DNA were found to resonate with $2.55-8.75 \mathrm{GHz}, 2.75-5.60 \mathrm{GHz}, 4.10 \mathrm{GHz}$, and $2.65 \mathrm{GHz}$ respectively [25]. This indicates that DNA polymer chain length determining the structural conformation and size (globular or linear, large or small) directly correspond to their respective resonant frequencies. Illustratively, a resonance shift occurs within the frequency range of $41-52 \mathrm{GHz}$ upon changing the length of the haploid genetic material in E. coli [26]. Further, relative viscosity measurements showed that the resonance frequencies decreased proportionally to the enhancement of haploid genome length. These reports suggest that resonant interactions of MMW with genetic material can arise by structure based energetic coupling without causing biochemical genomic alterations (ref. Fig. 2) within the threshold powers of non-thermal exposure. Experimentally, irradiation of transformed Human Corneal Epithelial (HCE-T) and Human Lens Epithelial (SRA01/04) cell lines by $60 \mathrm{GHz}$ at $1 \mathrm{~mW} / \mathrm{cm}^{2}$ over 24 hours of constant exposure found no statistically significant genotoxic effects on the nucleus [27].

Further, resonance absorption of MMW irradiation corresponding to different DNA polymer chain lengths [25] and found to be related to their structural conformation and size (globular or linear, large or small) as discussed above [ref. Results section 2.2]. Thus, certain irradiation regimes may exercise switch ON/Off control over genetic expression by affecting conformational changes which translate into specific modifications of the proteome determining the extent of cell division effects. Experimentally, resonance shifts occurring within the frequency range of 41-52 GHz corresponded to changing lengths of the genetic material in E. coli located directly in the cytosol of the cells [26]. In eukaryotes, the genetic material in the nucleus is distinguishable into heterochromatin (condensed and inactive) and euchromatin (actively directing protein synthesis). MMW irradiation regimes may be affecting conformational changes on chromatin which modulates the gene expression without causing genetic DNA damage. Such a mechanism can be analogous to the biochemical ON/OFF control of epigenetic regulation wherein specific biological molecules physically associate or dissociate with certain genetic sequences in the nucleus in response to external stimuli for either blocking or enhancing gene expression accordingly. In contrast, by involving a medium of radiation to enable non-biochemical control of genetic expression through conformational changes in the genome, MMW therapy can provide interesting applications of eliciting targeted biological responses for various purposes. Such effects are likely to be dose- dependent and potential applications of this technique will require characterization of the genomic conformational changes possible for different exposure conditions correspondent with their respective biological outcomes. Such a mechanism of action can also explain the ambiguous cell proliferation results after MMW treatment from the other studies described above in the introduction $[12,13,14]$.

A biological cell is a compartmentalized structure separated from the surrounding environment by the lipid cell membrane containing transmembrane proteins. It has been reported that $65 \mathrm{GHz}$ irradiation reduced the effects of factors on yeast cells due to the destabilization of intracellular water structure [28]. Under physiological conditions, yeast cells are reported to bear $65 \%$ water by composition with about $20-30 \%$ of the volume inhabited by cytosolic proteins that participate in the most metabolic processes of the cells [29]. Biological functions at the cellular level are affected by 
proteins, and the functionality of proteins is, in turn, determined by their molecular structure. Proteins are polypeptide chains composed of sequentially joined amino acids folding into the lowest energy conformations in their physiological environment giving rise to three-dimensional structures. These structures are essential for the protein's biochemical interactions with other molecules which manifests in biological functions. Changes in the cytosol environment can therefore translate into changing the properties of biomolecules like proteins by affecting their charge densities in space and time.

Interactions of MMW irradiation with proteins in the cytosol could generate structural and/or functional changes which subsequently affects cell functionality. Structurally, water is a physical participant during the folding of the polypeptide chain in protein folding through hydrophobic collapse [29]. Thus, water interacts with proteins to affect their dynamics. Conversely, changes in the chemical composition of the aqueous environment can alter the three-dimensional structure of proteins. Molecular Transfer Model (MTM) predicts conformational changes in protein structures when $\mathrm{pH}$ changes occur in the vicinity in solution using calculated partition functions of polypeptides [30]. Illustratively, Nitrophorin 4 (NP4) is a protein that releases nitric oxide (NO) in a $\mathrm{pH}$-sensitive manner. NP4 remains in a closed conformation and tightly binds $\mathrm{NO}$ at $\mathrm{pH} 5.5$ [31]. At $\mathrm{pH} 7.5$, deprotonation occurs, changing the conformation and releasing NO.

Since MMW are classed as non-ionizing radiation with low $0.3-0.4 \mathrm{meV}$ range of photon energies, their direct exposure is not expected to directly generate Reactive Oxygen Species (ROS) by biochemical degradation unlike ionizing radiation therapies [2, 3], which involve higher photon energies and thereby provide only safety restricted applications. We conjecture that MMW elicits therapeutic effects and limit the adversities by the phenomenon of Structure Resonant Energy Transfer (SRET). It allows membrane bound spherical core - shell charge separated bodies (from 28 - $100 \mathrm{~nm}$ diameters) to exhibit dipolar coupling with incident electric fields [32] in the range of 12 $45 \mathrm{GHz}$ of the electromagnetic spectrum with an inverse relation between frequency and size dimensions. Such phenomena which occur at resonant frequencies determined by the structural dimensions and charge status can lead to rupture events [33], resulting in leakage of matrix contents depending on the exposure power conditions and duration of treatment. Cells are composed of multiple subcellular organelles bound by membranes which maintain distinct environments within the matrix separated from the cytoplasm outside. Peroxisomes and microbodies are a class of cell organelles which function as a source of Reactive Oxygen Species (ROS). ROS causes oxidative stress and DNA damage at high levels inhibiting cell division [34]. Generally observed to be spherical in shape, peroxisomes resemble the membrane bound core-shell charge separated structures described above [32], raising the possibility that $85-105 \mathrm{GHz}$ MMW irradiation may have resulted in peroxisome rupture with subsequent ROS leakage leading to inhibited cell division. However, dipolar coupling at $85-105 \mathrm{GHz}$ frequencies will correspond to charge separated spherical membrane bound structures of diameters around $11-14 \mathrm{~nm}$ [32]; which are much below the peroxisomes size range of $200-500 \mathrm{~nm}[35,36]$, indicating that reduced cell division upon $85-105$ $\mathrm{GHz}$ exposure is not accountable to ROS mediated mechanisms. However, other smaller vesicles with resonant dimensions covered by this bandwidth may contribute to the observed non-thermal effects by different non-ROS mediated pathways. Such processes could explain the ambiguous MMW treatment results reported from other studies $[12,13,14]$ described above in the introduction.

As such, the apoptosis-promoting effects MMW radiation on several types of tumors could be also be a potential reason for the observed effects. Sweeping frequency regimes over $75-105 \mathrm{GHz}$ at $0.2 \mathrm{~mW} / \mathrm{cm}^{2}$ resulted in apoptosis of H1299 lung cancer cells [5]. Experimentally, irradiation at 35 $\mathrm{GHz}$ is reported to activate the apoptosis in A375 melanoma cells in vitro [38]. The transient receptor potential vanilloid 1 (TRPV1) expression is altered in prostate, colon, bladder, pancreas and breast cancers. TRPV1 forms high conductivity channels in the cell membrane, and its prolonged activation could lead to cell apoptosis. Pre-treatment with capsaicin, TRPV1 agonist and subsequent exposure to $60 \mathrm{GHz}$ radiation at $100 \mathrm{~mW}$ for $20 \mathrm{~min}$ facilitates apoptosis in Triple Negative Breast Cancer Cell Lines [39]. The combined action of TRPV1 agonist and MMW radiation demonstrates robust 
sensitization to cell death even at low concentrations of the chemical agonist. Our experiments have showed complete termination of the cells' growth at $17.17 \mathrm{~mW} / \mathrm{cm}^{2}$; possibly via strong apoptotic effect by using the higher power densities that was required for better penetration since yeast cells bear thick cell walls which were absent in the H1299 and A375 cells described above. However, further experiments are requisite to exclude intracellular thermal effects or responses of the cells when using an open - ended waveguide. We intend to investigate the complete range of effects of the W-band (85-105 GHz) MMW irradiation to unravel the mechanism(s) of action by using deleted yeast library collections of over 5100 single gene deletions.

In summary, our experiments examined the effects of MMW (85-105 GHz) on Saccharomyces Cerevisiae yeast and demonstrate that W-band ( $85-105 \mathrm{GHz}) \mathrm{MMW}$ irradiation at a power density of $\sim 1.0 \mathrm{~mW} / \mathrm{cm}^{2}$ for $5-6$ hours exposure duration results in a $62 \%$ reduction in the growth rate of irradiated yeast cells at a density of 50 cells/ $\mu$ l. MMW radiation effects were found to be frequency, energy dose and cell density dependent. Experimental exposure conditions maintained within the ICNIRP (International Commission on Non-Ionizing Radiation Protection) non-thermal safety standard of $20 \mathrm{~mW} / \mathrm{cm}^{2}$ [40]. Further, measurements of temperature rise demonstrated that effects were non-thermal in nature. A comparative analysis of changes in the growth profile of irradiated wild type and $\Delta$ rad52 (DNA damage repair) deletion cells revealed no directed detrimental effects on genomic stability arising from this non-ionizing radiation treatment. Analyses of dose dependent effects of MMW propagation and calibrations of cell density to exposure conditions revealed threshold parameters which can be used for targeted inhibition of infectious microbes. Specifically, using an open - ended waveguide delivering a higher power density of $17.17 \mathrm{~mW} / \mathrm{cm}^{2}$ for $3-4$ hours exposure duration achieved complete termination of cellular proliferation in $50 \mathrm{cells} / \mu \mathrm{l}$ samples of Saccharomyces Cerevisiae yeast cells.

Our results indicate that MMW irradiation can be useful for future application in medical treatments. The advantages in using non-ionizing MMW radiation enabling non-thermal control of targeted biological responses with or without chemical and biological drugs has promising potential for innovative biomedical procedures and devices.

Invasive fungal infections remain a serious clinical problem. The current use of several classes of antifungal drugs have led to increased antimicrobial resistance of pathological species like $C$. albicans by accelerating the development of mutations, overexpression of multidrug efflux pumps [41], etc. Moreover, C. albicans as a pathogen depending largely on its ability to generate diversity at not only the genomic but also the morphological and physiological levels by its ability to switch from yeast to hyphal or pseudo hyphal forms [42, 43]; constitutes a major challenge in the development of new anti-fungal drugs and therapeutic strategies. A direct application of the MMW radiation effect mechanisms presented in this article for non-thermal exposure regimes can be used for treating pathogenic fungal infections (via cell division inhibition effect, ref. Fig 1) which are common in dermatology [44] and reproductive health [45]; and cancer lesion/tissue treatments (via cancer cell mortality effect $[4,5]$ ) by appropriately scaling the exposure conditions described here to match clinical cell densities; besides various other promising purposes. Further, recent advances in endoscopic probes [46] operating in the Super High Frequency (SHF) $3-30 \mathrm{GHz}$ bandwidths and similar technological trends will enable novel avenues of adapting such non-thermal techniques to treat internal body sites overcoming the burn - hazard restrictions in application of conventional thermal ablation procedures. Our experiments demonstrate that non-thermal regimes of nonionizing (85-105 GHz) MMW exposure have a promising potential not only for anti-cancer treatments $[4,5]$, but for targeted treatment of yeast related diseases.

\section{Materials and Methods}

\subsection{Conditions of Cell Culture}

Wild type (WT) budding yeast BY4741 Saccharomyces cerevisiae (MATa his3 $\Delta 1$ leu2 $\Delta 0$ met15 $\Delta 0$ ura3 $\Delta 0$ ) and its $\Delta$ rad52 mutant strain BY4741 Saccharomyces cerevisiae (MATa his3 $\Delta 1$ leu2 $\Delta 0$ met15 $\Delta 0$ ura3 $\triangle 0$ RAD52::KanMX4) available through EUROSCARF (Frankfurt, Germany) were used for 
irradiation. Cells were grown in standard synthetic complete (SC) agar and liquid mediums at a temperature of $30^{\circ} \mathrm{C}$. The growth rate of both control and irradiated cells were measured using an absorbance plot at $600 \mathrm{~nm}$ measured by a standard spectrophotometer in units of optical density (OD). Cultures were adequately diluted to 0.1 OD using a standard absorbance plot at the start of experiment and incubated until they reached an OD value of 0.4 (the point at which cells initiate the logarithmic growth phase). Cultures at 0.4 OD were diluted to 10000, 1000, 100 and 50 cells/ $\mu$ l (to determine the optimal concentration of cells and energy dosage). 1-2 $\mu$ l volume of those solutions were dropped onto SC agar plates. Six colonies were seeded in two replicates: one for irradiation and another for comparison as control (sham) for each discrete frequency regime. After irradiation, the cells were transferred to SC liquid medium and incubated under standard conditions. Growth rate of both irradiated and control (sham) yeast cells were measured regularly at intervals of 90 minutes over a period of $\sim 8 \mathrm{~h}$ to assay the effect of MMW exposure on physiological growth.

\subsection{Conditions of Irradiation}

The schematic diagram of the experimental setup for MMW irradiation is illustrated in Fig. 5. Yeast cells were spotted on the Agar medium prior to irradiation. The agar layer thickness was kept constant throughout ( $2 \mathrm{ml}$ volume) on $35 \mathrm{~mm}$ polystyrene plates . Cells were exposed to specific frequencies of $85 \mathrm{GHz}, 95 \mathrm{GHz}$, and $105 \mathrm{GHz}$ at $5 \mathrm{dBm}$ power for 6 hours respectively. The irradiation experiment for each frequency involved six distinct experiments at the same cell density. MMW (85$110 \mathrm{GHz}$ ) were generated using a signal generator (Keysight technology, N5183B, 9 kHz-20 GHz), and 6× active frequency multiplier (Quinstar Tech Inc., QMM-311220025). A standard gain pyramidal horn antenna (Quinstar Tech Inc., QWH-WPRROO) was used for MMW emission. The power of transmitted waves from the horn was measured using an identical horn antenna and digital storage oscilloscope (Agilent technology, DSO-X 2004A). Distribution of relative energy across antenna aperture was measured using an open-ended waveguide (Quinstar Tech Inc., QWH-WPRROO) in the near field (ref. Fig. 6).

SAR (Specific Absorption Rate) was calculated (Table 1, see in results). It was in range 3.48-2.08 $\mathrm{W} / \mathrm{kg}$ involving the horn antenna and $43.05 \mathrm{~W} / \mathrm{kg}$ for the irradiations with $85 \mathrm{GHz}$ using the waveguide respectively. $S A R=\frac{P_{a}}{m}=\frac{P_{a}}{\rho V}=\frac{P_{a} / S}{\rho l}$ was used to calculate the SAR values where $S A R, P_{a}$, $m, \rho, V, S$, and $l$ denote. Specific Absorption Rate, absorbed power, mass, density, volume, surface area and depth of medium irradiated respectively. As most of the radiation was probably absorbed by the agar rather than the cells, the total volume is more relevant than dry cell mass. Density $\rho$ was considered approximately equal to water $\left(997 \mathrm{~kg} / \mathrm{m}^{3}\right)$ and depth $l \mathrm{used}$ in the experiments was $4 \mathrm{~mm}$. Cells were irradiated after being immersed on the agar.

The penetration depth of $85-110 \mathrm{GHz}$ MMW is very shallow, hence the exposed surface area and not the volume is considered. The appropriate exposure metric for MMW is therefore the power density, power per area $\left(\mathrm{W} / \mathrm{m}^{2}\right)$. Absorbed power $\mathrm{P}_{\mathrm{a}}$ was calculated as $P_{a}=$ Incident Power Reflected Power. $P_{a} / \mathrm{s}$-represents the power densities of the irradiations used $\left(1.39 \mathrm{~mW} / \mathrm{cm}^{2}\right.$ for horn antenna and $17.17 \mathrm{~mW} / \mathrm{cm}^{2}$ for the wave guide). 

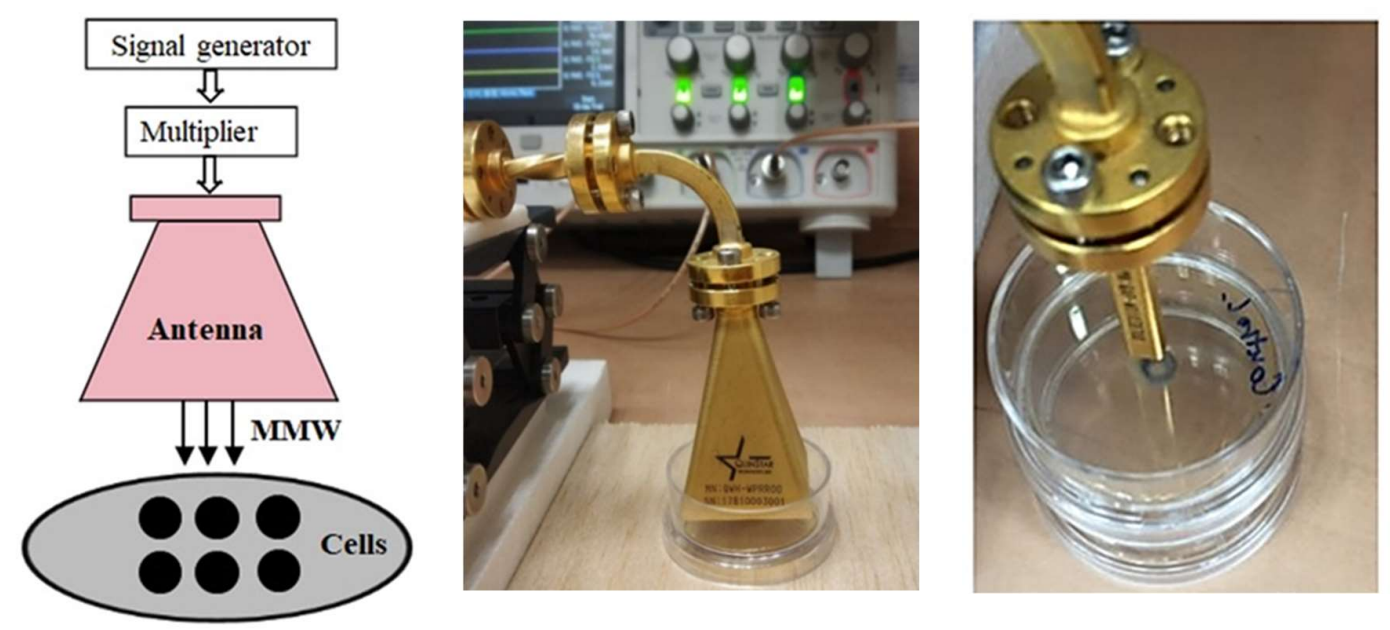

Figure 5. Block diagram of the experimental setup for irradiation and pictures of the pyramidal horn antenna and wave guide.

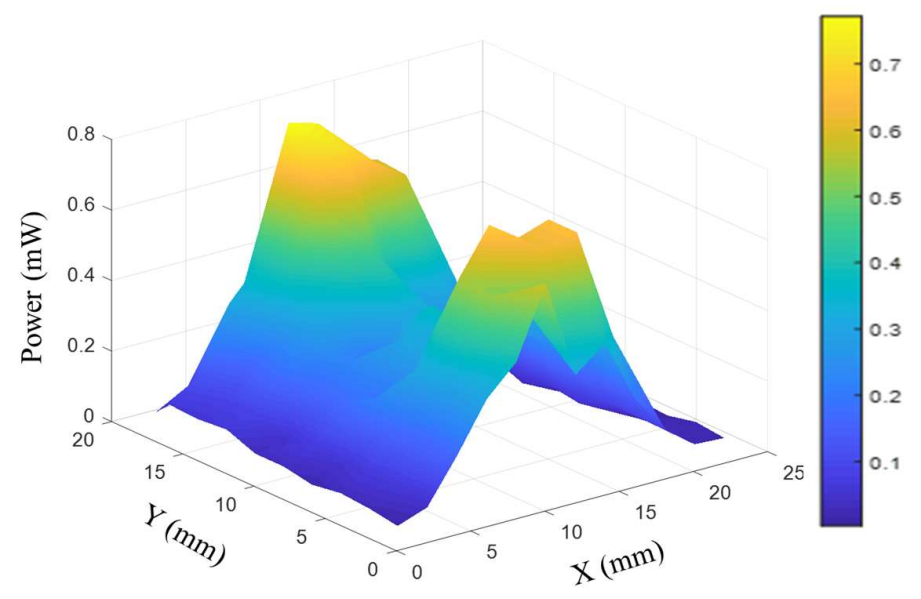

Figure 6. Relative distribution of power across horn antenna aperture.

Author Contributions: Conceptualization, A.B. and S.L-A.; methodology, A.B., S.R. and S.L-A.; software, A.B, S.R., K.K. and S.L-A.; validation, S.L-A., K.K., J.L. and A.Y.; formal analysis, A.B., S.R., S.L-A. and A.Y.; investigation, A.B. and S.R.; resources, S.L-A., K.K., J.L., and A.Y.; data curation, A.B., S.R. and S.L-A.; writingoriginal draft preparation, A.B.; S.R., S.L.-A, writing-review and editing, A.B., S.R., S.L-A. K.K., and A.Y.; visualization, A.B., S.R. and S.L-A.; supervision, S.L-A.; project administration, S.L-A. and A.Y.; funding acquisition, S.L-A., K.K., J.L. and A.Y. All authors have read and agreed to the published version of the manuscript.

Funding: This research was funded by the Ariel Center for Applied Cancer Research

Acknowledgments: The authors acknowledge the Israeli Council for Higher Education (CHE) for fellowship.

Conflicts of Interest: The authors declare no conflicts of interest.

\section{References}


1. Koo, M. M.; Swann, R.; Mcphail, S.; Abel, G. A.; Elliss-Brookes, L.; Rubin, G. P.; Lyratzopoulos, G. Presenting Symptoms of Cancer and Stage at Diagnosis: Evidence from a Cross-Sectional, PopulationBased Study. The Lancet Oncology 2020, 21 (1), 73-79.

2. Ryan, J. L. Ionizing Radiation: The Good, the Bad, and the Ugly. Journal of Investigative Dermatology 2012, 132 (3), 985-993.

3. Angelis, C. D.; Salvo, N.; Barnes, E.; Draanen, J. V.; Stacey, E.; Mitera, G.; Breen, D.; Giotis, A.; Czarnota, G.; Pang, J. Prophylaxis and Management of Acute Radiation-Induced Skin Reactions: a Systematic Review of the Literature. Current Oncology 2010, 17 (4).

4. Komoshvili, K.; Becker, T.; Levitan, J.; Yahalom, A.; Barbora, A.; Liberman-Aronov, S. Morphological Changes in H1299 Human Lung Cancer Cells Following W-Band Millimeter-Wave Irradiation. Applied Sciences 2020, $10(9), 3187$.

5. Komoshvili, K.; Israel, K.; Levitan, J.; Yahalom, A.; Barbora, A.; Liberman-Aronov, S. W-Band Millimeter Waves Targeted Mortality of H1299 Human Lung Cancer Cells without Affecting NonTumorigenic MCF-10A Human Epithelial Cells In Vitro. Applied Sciences 2020, 10 (14), 4813.

6. Mirbeik-Sabzevari, A.; Tavassolian, N. Ultrawideband, Stable Normal and Cancer Skin Tissue Phantoms for Millimeter-Wave Skin Cancer Imaging. IEEE Transactions on Biomedical Engineering 2019, $66(1), 176-186$.

7. Yu, G.; Coln, E.; Schoenbach, K.; Gellerman, M.; Fox, P.; Rec, L.; Beebe, S.; Liu, S. A Study on Biological Effects of Low-Intensity Millimeter Waves. IEEE Transactions on Plasma Science 2002, 30 (4), 1489-1496.

8. Ziskin, M. C. Physiological Mechanisms Underlying Millimeter Wave Therapy. BIOELECTROMAGNETICS Current Concepts 2006, 241-251.

9. Altmann, K.; Dürr, M.; Westermann, B. Saccharomyces Cerevisiae as a Model Organism to Study Mitochondrial Biology. Methods in Molecular Biology Mitochondria 2007, 81-90.

10. Beck, H.; Dobritzsch, D.; Piåjkur, J. Saccharomyces Kluyverias a Model Organism to Study Pyrimidine Degradation. FEMS Yeast Research 2008, 8 (8), 1209-1213.

11. Kachroo, A. H.; Laurent, J. M.; Yellman, C. M.; Meyer, A. G.; Wilke, C. O.; Marcotte, E. M. Systematic Humanization of Yeast Genes Reveals Conserved Functions and Genetic Modularity. Science 2015, 348 (6237), 921-925.

12. Grundler, W. Intensity- and Frequency-Dependent Effects of Microwaves on Cell Growth Rates. Journal of Electroanalytical Chemistry 1992, 342 (3), 361-365.

13. Grundler, W.; Keilmann, F.; Fröhlich, H. Resonant Growth Rate Response of Yeast Cells Irradiated by Weak Microwaves. Physics Letters A 1977, 62 (6), 463-466.

14. Furia, L.; Hill, D. W.; Gandhi, O. P. Effect of Millimeter-Wave Irradiation on Growth of Saccharomyces Cerevisiae. IEEE Transactions on Biomedical Engineering 1986, BME-33 (11), 993-999.

15. Vojisavljevic, V.; Alsuhaim, H. S.; Pirogova, E. Low power microwave exposures at $968 \mathrm{MHz}$ increase the growth rate of Breanomyces bruxellensis yeast cells. 2016 IEEE International Conference on Microwave and Millimeter Wave Technology (ICMMT), Beijing, 2016, pp. 1061-1063.

16. Smolyanskaya, A. Z.; Vilenskaya, R. L. Effects of Millimeter-Band Electromagnetic Radiation on the Functional Activity of Certain Genetic Elements of Bacterial Cells. Soviet Physics Uspekhi 1974, 16 (4), 571-572.

17. K. Komoshvili, J. Levitan, S. Aronov, B. Kapilevich and A. Yahalom. Millimeter waves non-thermal effect on human lung cancer cells. 2011 IEEE International Conference on Microwaves, Communications, Antennas and Electronic Systems (COMCAS 2011), Tel Aviv, 2011, pp. 
18. Feng $Z$, Scott SP, Bussen $W$, et al. Rad52 inactivation is synthetically lethal with BRCA2 deficiency. Proc Natl Acad Sci U S A. 2011;108(2):686-691.

19. Bhowmick R, Minocherhomji S, Hickson ID. RAD52 Facilitates Mitotic DNA Synthesis Following Replication Stress. Mol Cell. 2016;64(6):1117-1126.

20. Yasuhara T, Kato R, Hagiwara Y, et al. Human Rad52 Promotes XPG-Mediated R-loop Processing to Initiate Transcription-Associated Homologous Recombination Repair. Cell. 2018;175(2):558-570.e11.

21. Game, J. C. Radiation-Sensitive Mutants and Repair in Yeast. Springer Series in Molecular Biology Yeast Genetics 1983, 109-137.

22. Game JC. DNA double-strand breaks and the RAD50-RAD57 genes in Saccharomyces. Semin Cancer Biol. 1993;4(2):73-83.

23. Lunkenheimer, P.; Emmert, S.; Gulich, R.; Köhler, M.; Wolf, M.; Schwab, M.; Loidl, A. ElectromagneticRadiation Absorption by Water. Physical Review E 2017, 96 (6).

24. White, J. Variation In Water Content Of Yeast Cells Caused By Varying Temperatures Of Growth And By Other Cultural Conditions. Journal of the Institute of Brewing 1952, 58 (1), 47-50.

25. Edwards, G. S.; Davis, C. C.; Saffer, J. D.; Swicord, M. L. Resonant Microwave Absorption of Selected DNA Molecules. Physical Review Letters 1984, 53 (21), 2060-2060.

26. Belyaev, I. Y.; Alipov, Y. D.; Polunin, V. A.; Shcheglov, V. S. Evidence for Dependence of Resonant Frequency of Millimeter Wave Interaction With Escherichia coliK12 Cells on Haploid Genome Length. Electro- and Magnetobiology 1993, 12 (1), 39-49.

27. Koyama, S.; Narita, E.; Shimizu, Y.; Suzuki, Y.; Shiina, T.; Taki, M.; Shinohara, N.; Miyakoshi, J. Effects of Long-Term Exposure to $60 \mathrm{GHz}$ Millimeter-Wavelength Radiation on the Genotoxicity and Heat Shock Protein (Hsp) Expression of Cells Derived from Human Eye. International Journal of Environmental Research and Public Health 2016, 13 (8), 802.

28. Svetlana M. Rogacheva, Milena I. Babaeva. The effect of millimeter waves at the yeast Saccharomyces cerevisiae during heliogeophysical disturbances. Proc. SPIE 8699, Saratov Fall Meeting 2012: Optical Technologies in Biophysics and Medicine XIV; and Laser Physics and Photonics XIV, 86990N.

29. Bellissent-Funel, M.-C.; Hassanali, A.; Havenith, M.; Henchman, R.; Pohl, P.; Sterpone, F.; Spoel, D. V. D.; Xu, Y.; Garcia, A. E. Water Determines the Structure and Dynamics of Proteins. Chemical Reviews 2016, 116 (13), 7673-7697.

30. O'Brien, E. P.; Brooks, B. R.; Thirumalai, D. Effects of PH on Proteins: Predictions for Ensemble and Single-Molecule Pulling Experiments. Journal of the American Chemical Society 2011, 134 (2), 979-987.

31. Russo, N. V. D.; Estrin, D. A.; Martí, M. A.; Roitberg, A. E. PH-Dependent Conformational Changes in Proteins and Their Effect on Experimental PKas: The Case of Nitrophorin 4. PLoS Computational Biology 2012, 8 (11).

32. Liu, T.-M.; Chen, H.-P.; Wang, L.-T.; Wang, J.-R.; Luo, T.-N.; Chen, Y.-J.; Liu, S.-I.; Sun, C.-K. Microwave Resonant Absorption of Viruses through Dipolar Coupling with Confined Acoustic Vibrations. Applied Physics Letters 2009, 94 (4), 043902.

33. Yang, S.-C.; Lin, H.-C.; Liu, T.-M.; Lu, J.-T.; Hung, W.-T.; Huang, Y.-R.; Tsai, Y.-C.; Kao, C.-L.; Chen, S.Y.; Sun, C.-K. Efficient Structure Resonance Energy Transfer from Microwaves to Confined Acoustic Vibrations in Viruses. Scientific Reports 2015, 5 (1).

34. Havens C, Ho A, Yoshioka N, Dowdy S. Regulation of Late G1/S Phase Transition and APCCdh1 by Reactive Oxygen Species. Molecular and Cellular Biology 2006, 26 (12) 4701-4711. 
35. Veenhuis M, Mateblowski M, Kunau WH, Harder W. Proliferation of microbodies in Saccharomyces cerevisiae. Yeast. 1987;3(2):77-84.

36. Yofe I, Soliman K, Chuartzman SG, et al. Pex35 is a regulator of peroxisome abundance. J Cell Sci. 2017;130(4):791-804.

37. Deng $\mathrm{X}$, Deng $\mathrm{T}, \mathrm{Ni} \mathrm{Y}$, et al. Cytochrome $c$ modulates the mitochondrial signaling pathway and polymorphonuclear neutrophil apoptosis in bile duct-ligated rats. Exp Ther Med. 2016;12(1):333-342. doi:10.3892/etm.2016.3313

38. Zhao, R.; Liu, Y.; Liu, S.; Luo, T.; Zhong, G.; Liu, A.; Zeng, Q.; Xin, X. Millimeter wave exposure induces apoptosis in human melanoma A375 cells in vitro. Nan Fang Yi Ke Da Xue Xue Bao 2019, 39, 76-81.

39. A. Sorolla, S. Romanenko, P. H. Siegel and V. Wallace, "Utilisation of MMW Radiation to Facilitate Apoptosis in Triple Negative Breast Cancer Cell Lines via TRPV1 Receptor Sensitization," 2019 44th International Conference on Infrared, Millimeter, and Terahertz Waves (IRMMW-THz), Paris, France, 2019, pp. 1-2, doi: 10.1109/IRMMW-THz.2019.8873881.

40. ICNIRP. Guidelines for Limiting Exposure to Electromagnetic Fields (100 kHz to $300 \mathrm{GHz}$ ). Health Physics. 2020;118(5):483-524.

41. Cowen, L.E.; Steinbach, W.J. Stress, drugs, and evolution: The role of cellular signaling in fungal drug resistance. Eukaryot. Cell 2008, 7, 747-764.

42. Miranda, I.; Rocha, R.; Santos, M.C.; Mateus, D.D.; Moura, G.R.; Carreto, L.; Santos, M.A. A genetic code alteration is a phenotype diversity generator in the human pathogen Candida albicans. PLoS ONE 2007, 2, e996.

43. Silva, R.M.; Paredes, J.A.; Moura, G.R.; Manadas, B.; Lima-Costa, T.; Rocha, R.; Miranda, I.; Gomes, A.C.; Koerkamp, M.J.; Perrot, M.; et al. Critical roles for a genetic code alteration in the evolution of the genus Candida. EMBO J. 2007, 26, 4555-4565.

44. Vázquez-González D, Perusquía-Ortiz AM, Hundeiker M, Bonifaz A. Opportunistic yeast infections: candidiasis, cryptococcosis, trichosporonosis and geotrichosis. J Dtsch Dermatol Ges. 2013;11(5):381-394.

45. Aubyn GB, Tagoe DNA. Prevalence of vaginal infections and associated lifestyles of students in the university of Cape Coast, Ghana. Asian Pac J Trop Dis. 2013;3(4):267-270. doi:10.1016/S22221808(13)60068-7

46. Hancock C. SUMCASTEC_180121_NA_IMS17WSPresentation_Workshop IMS Conference paper.pdf_Bath_C. Hancock_Partners and public_Last draft. Zenodo. https://zenodo.org/record/1156362. Published January 21, 2018. 